DOI: 10.12731/2227-930X-2019-4-27-31

УДК 656

\title{
ОСОБЕННОСТИ МОДЕЛИРОВАНИЯ ЛОГИСТИЧЕСКИХ СИСТЕМ
}

\section{Шакиров А.А., Зарипова Р.С.}

Целью данной статьи является изучение того, насколько важно учитывать неопределенность в проблеме моделирования логистической системы на этапе планирования. Рассмотрены структурные различия мезжду решением, основанным на неопределенном спросе, и решением, основанным на детерминированном спросе. Некоторые из этих различий помогают хеджировать неопределенность, не увеличивая при этом стоимость эксплуатащии графика.

Ключевые слова: моделирование; логистическая система; грузоперевозки; логистика.

\section{FEATURES OF MODELING OF THE LOGISTICS SYSTEM}

\section{Shakirov A.A., Zaripova R.S.}

The purpose of this article is to study how important it is to take into account the uncertainty in the problem of designing a logistics system at the planning stage. The structural differences between a solution based on uncertain demand and a solution based on deterministic demand are considered. Some of these differences help hedge uncertainty without increasing the cost of operating the chart.

Keywords: modeling; logistics system; cargo transportation; logistics.

Целью проектирования логистической системы является определение маршрутов, по которым будет предлагаться услуга, тип услуги, которая будет предлагаться, а также периодичность рас- 
писания. Выбранные услуги и расписание сгруппированы в план транспортировки или загрузки. Расписание также публикуется в интересах потенциальных пользователей системы. При построении плана необходимо стремиться к эффективной работе с точки зрения общей стоимости системы, учитывая доступные ресурсы, известные требования и уровень качества обслуживания, которого перевозчик желает достичь.

Существует много дискуссий о важности перехода от детерминированных к стохастическим моделям. Проблемы очевидны в ранних работах Данцига и Фергюсона, а также в более поздних работах, Уоллеса и Хигла. К сожалению, это относится и к большей части работы, выполняемой в проектировании системы, которая почти всегда выполнялась детерминистическим образом. Тогда можно спросить: что теряется, если не интегрировать информацию, касающуюся стохастической природы спроса, в методологию тактического планирования? Приведет ли интеграция такой информации к различным шаблонам проектирования услуг, либо в выбранных услугах, либо в используемых стратегиях консолидации? Будут ли итоговые план и график транспортировки более надежными, чем те, которые мы в настоящее время получаем в отношении повседневных операций?

Цель этой статьи - помочь ответить на эти вопросы. Они имеют отношение к ситуациям, когда используются детерминированные модели оптимизации в тех случаях, когда стохастика явно присутствует. Мы должны спросить: приведет ли решение детерминированной модели, а не стохастической, к существенным ошибкам или мы просто говорим о незначительных корректировках? Вычислительные, а также моделирующие усилия, необходимые для решения стохастического случая, существенны, поэтому мы должны быть уверены, что оно того стоит. Или, по крайней мере, что потенциальные ошибки должны быть такими, что проверка фактических эффектов должна стоить затрат. Решения стохастических моделей более гибки в отношении неопределенности, чем решения для детерминированных моделей. Под гибкостью под- 
разумеваем, что они более экономично справляются со многими различными требованиями, что приводит к снижению ожидаемых затрат [1].

Целью данной статьи является исследование того, как две модели ведут себя по отношению друг к другу. Первое важное наблюдение заключается в том, что ожидаемое поведение решения, полученного из детерминированной модели, может быть произвольно хуже, чем из стохастической модели. Решения стохастических моделей структурно отличаются от решений детерминированных моделей. Планирование для реальных операторов ГМГ или для других способов консолидации может быть выполнено с использованием различных методов, например, надежной оптимизации, создания планов.

Чтобы начать изучение влияния введения стохастических элементов в формулировки проектирования систем, возьмем упрощенную версию проблемы, с которой сталкиваются операторы ГМГ, в которой периодические графики составляются для ряда транспортных средств и где только спрос может стохастически меняться. Результаты показывают, что интеграция стохастических элементов в модель проектирования системы выгодна, и результирующий план транспортировки является более гибким. Предложенная модель стохастической системы принимает форму детерминированных фиксированных затрат, емкостных формулировок проектирования многокомпонентных систем. Затраты, которые варьируются в зависимости от интенсивности обслуживания и движения товаров, связаны с движением товаров и услуг. Цель состоит в том, чтобы минимизировать стоимость системы или максимизировать прибыль при ограничениях, обеспечивающих правила и цели спроса, обслуживания и эксплуатации.

Для исследования была создана простая версия модели проектирования многопериодной системы, основанной на кейсе для автомобильного перевозчика с меньшей загрузкой. Было сделано несколько упрощающих предположений: рассмотрен однородный парк транспортных средств без ограничений на количество 
используемых транспортных средств; транспортные перевозки требуют одного периода, в то время как терминальные операции происходят мгновенно (в течение периода); требование не может быть доставлено позднее срока оплаты, но может быть получено раньше; существует фиксированная стоимость, связанная с эксплуатацией транспортного средства услуги, но никакие расходы не связаны с перемещением груза, то есть перемещения грузовиков стоят одинаково, перемещаются ли они загруженными или пустыми; нет затрат, связанных с задержками или операциями терминала; план повторяется периодически. Полученная модель достаточно проста, чтобы ее можно решить с помощью коммерческого готового смешанного целого программного обеспечения. С другой стороны, проблема сохраняет основные характеристики проекта сети обслуживания и, таким образом, предлагает хорошие условия для изучения явного рассмотрения стохастики.

\section{Список литературы}

1. Шакиров А.А. Трансформация систем учета и контроля в условиях цифровой экономики / А.А. Шакиров, Р.С. Зарипова // Наука Красноярья. 2019. Т. 8. № 3-2. С. 112-115.

\section{References}

1. Shakirov A.A. Transformaciya sistem ucheta i kontrolya v usloviyah cifrovoj ekonomiki / A.A. SHakirov, R.S. Zaripova // Nauka Krasnoyar’ya. 2019. T. 8. № 3-2. S. 112-115.

\section{ДАННЫЕ ОБ АВТОРАХ}

Шакиров Арслан Айнурович, студент

Казанский государственный энергетический университет ул. Красносельская, 51, г. Казань, 420066, Российская Федеращия

Зарипова Римма Солтановна, доцент, канд. техн. наук Казанский государственный энергетический университет ул. Красносельская, 51, г. Казань, 420066, Российская Федераџия zarim@rambler.ru 


\section{DATA ABOUT THE AUTHORS}

Shakirov Arslan Ainurovich, Student

Kazan State Power Engineering University

51, Krasnoselskaya str., Kazan, 420066, Russian Federation

Zaripova Rimma Soltanovna, Associate Professor, Candidate of Technical Sciences

Kazan State Power Engineering University

51, Krasnoselskaya str., Kazan, 420066, Russian Federation zarim@rambler.ru 